\title{
Etude descriptive de la formation d'acrylamide dans quelques aliments à risques industriels et domestiques en Côte d'Ivoire
}

\author{
Godi Henri Marius BIEGO ${ }^{1,2^{*}}$, Beli Eric SIKA ${ }^{1}$, Pierre EZOUA ${ }^{2}$, \\ Agbaya Serge Stéphane OGA ${ }^{1}$ et Kouakou Luc KOUADIO ${ }^{1}$ \\ ${ }^{I}$ Département de Santé Publique, d'Hydrologie et de Toxicologie, UFR des Sciences Pharmaceutiques et \\ Biologiques, Université de Cocody. BP V 34 Abidjan, Côte d'Ivoire. \\ ${ }^{2}$ Laboratoire de Biochimie et Sciences des Aliments, UFR Biosciences, Université de Cocody-Abidjan, 2 BP \\ 582 Abidjan, Côte d'Ivoire. \\ * Auteur correspondant, E-mail: biegoh3@yahoo.fr, Tél/Fax: (225) 212560 43, Mobile: (225) 07018836
}

\section{RESUME}

Les risques de formation de l'acrylamide dans certains aliments domestiques (pomme de terre, igname, banane plantain) et industriels dérivés du café et du cacao, consommés en Côte d'Ivoire, ont été évalués. Cette étude a été réalisée à partir d'une enquête descriptive des méthodes de fabrication, des modes et des conditions de préparation des aliments dans des structures industrielles, de formation ou de recherches, ainsi que dans des lieux de restauration collective. Les principaux facteurs de risque de formation in situ de l'acrylamide alimentaire sont la température (supérieure à $120^{\circ} \mathrm{C}$ ) et la durée des traitements thermiques industriels (torréfaction) ou domestiques (friture, grillade, cuisson au four). Ces risques sont plus élevés pour les frites de pomme de terre et pour les dérivés du café. L'analyse des méthodes de transformations industrielles et domestiques des aliments peut permettre de déterminer des indicateurs pour mettre en ouvre des mesures de prévention ou de réduction du risque de formation de l'acrylamide alimentaire.

() 2009 International Formulae Group. All rights reserved.

Mots-clés: Acrylamide, aliments, facteurs de risque.

\section{INTRODUCTION}

L'acrylamide est un composé obtenu par hydratation de l'acétonitrile en présence de catalyseurs inorganiques (manganèse, rhodium, cobalt) ou de biocatalyseur. Il est utilisé dans le traitement des eaux de consommation, des eaux usées industrielles, dans la production de gels d'électrophorèse, les industries pétrolières et cosmétiques, la construction et la fabrication de matières plastiques à usage alimentaire (FAO/WHO, 2002).

Ce composé a également une origine alimentaire et se forme dans certains aliments, à base d'amidon, cuits au four, frits, grillés ou rôtis (FAO/WHO, 2002; ONO et al., 2003).
Ces aliments sont issus, d'une part, des transformations industrielles (café, poudre de cacao, chocolat, biscuits, biscottes, céréales pour petit-déjeuner, pétales de maïs) et, d'autre part, des préparations domestiques (frites et chips de pomme de terre, chips de manioc, pain grillé, patate douce, pâtisserie, pop corn) (Rosen et Hellennas, 2002; ONO et al., 2003). Dans ces aliments, l'acrylamide se forme au cours de la réaction de Maillard en présence de glucose, fructose et asparagine, à une température supérieure à $120^{\circ} \mathrm{C}$ (Becalski et al., 2003; Stalder et al., 2003; Eriksson et al., 2007).

La toxicité de l'acrylamide a été révélée récemment chez plusieurs espèces 
animales où il serait neurotoxique (Edwards, 1976; Miller et al., 1982 ; Johnson et al., 1986), génotoxique (Shiraishi, 1978; Bull et al., 1984; IARC, 1994), tératogène (Smith et al., 1986; Marchetti et al., 1997) et carcinogène (Hashimoto et Aludridge, 1970; IARC, 1994). Son caractère neurotoxique a été également montré chez l'homme dans des études épidémiologiques réalisées en milieu professionnel (Bergmark et al., 1993; Bergmark, 1997). Les données existantes concernent surtout les pays industrialisés où l'exposition moyenne de la population a été estimée entre $0,3 \mu \mathrm{gg} / \mathrm{kg} / \mathrm{j}$ et $0,8 \mu \mathrm{gg} / \mathrm{kg} / \mathrm{j}$ (European Commision, 2002).

Les conditions de fabrication industrielle ou les modes de préparations domestiques de certains aliments, peuvent conduire à la formation in situ d'acrylamide alimentaire. Evaluer les risques liés à la transformation des denrées alimentaires proposées aux consommateurs ivoiriens permettrait de mettre en œuvre des stratégies de prévention, d'information, de sensibilisation et de réglementation afin de fournir des aliments de meilleure qualité. Par conséquence, notre étude a consisté à analyser les méthodes de fabrication des aliments dérivés du café et du cacao, ainsi que certains modes de préparations domestiques des aliments.

\section{MATERIEL ET METHODES \\ Matériel}

Le matériel d'étude est constitué des aliments industriels dérivés du café et du cacao et des aliments du cru tels que la pomme de terre, l'igname, la banane plantain, la patate douce, le maïs et les gâteaux (Tableaux 1 et 2). Ces aliments ont été enquêtés, d'une part, dans trois structures industrielles, un établissement de formation professionnelle et un centre de recherche et, d'autre part, dans des lieux de restauration collective.

\section{Méthodes}

Les données relatives aux aliments étudiés ont été collectées grâce à une enquête descriptive par questionnaire. L'évaluation du risque de formation de l'acrylamide a été réalisée par l'analyse des méthodes de fabrication des aliments dérivés du café et du cacao, et des modes et conditions de préparation artisanales des aliments du cru.

Tableau 1: Différentes étapes du traitement des fèves de cacao pour l'obtention de la pâte de cacao.

\begin{tabular}{|c|c|c|}
\hline Etapes & Technique & Conséquences et intérêts \\
\hline \multirow{3}{*}{$\begin{array}{l}1 \text { Torréfaction } \\
\text { (figure 1) }\end{array}$} & $\begin{array}{l}\text { Chauffage à } 110-132{ }^{\circ} \mathrm{C} \text { avant ou } \\
\text { après le décorticage }\end{array}$ & $\begin{array}{l}\text { Réaction de Maillard: développement des } \\
\text { arômes baisse de l'Index Chimique (IC) }\end{array}$ \\
\hline & Durée: 10 à 60 minutes & $\begin{array}{l}\text { (Formation à partir de la leucine et de la } \\
\text { phénylalanine de 5-méthyl-3-phényl-hexanal } \\
\text { à flaveur chocolatée) }\end{array}$ \\
\hline & & Séchage du grain pour permettre le broyage \\
\hline 2 Concassage & Eclatement des fèves dans un & Séparation de la coque et de l'amande \\
\hline ou Décorticage & concasseur & Dégermage: obtention du cacao pur \\
\hline \multirow[t]{6}{*}{3 Broyage } & Réduction des particules de fèves & Libération de la matière grasse \\
\hline & $\begin{array}{l}\text { issues des concasseurs par des } \\
\text { broyeuses (cylindres tournant à }\end{array}$ & Dispersion des débris végétaux \\
\hline & vitesse différentielle) & Obtention d'une Pâte de cacao \\
\hline & & Deux filières sont possibles: \\
\hline & & - $\quad$ poudre de cacao \\
\hline & & - chocolat \\
\hline
\end{tabular}


Tableau 2: Etapes du traitement des fèves de cacao pour l'obtention de la poudre de cacao et du chocolat.

\begin{tabular}{|c|c|c|}
\hline \multicolumn{3}{|c|}{ Filière 1 : Obtention de la poudre de cacao } \\
\hline Etapes & Technique & Conséquences et intérêts \\
\hline $\begin{array}{l}4 \text { Pressage et } \\
\text { extraction }\end{array}$ & $\begin{array}{l}\text { La pâte passe dans des } \\
\text { presses hydrauliques: } \\
\text { extraction par pression }\end{array}$ & $\begin{array}{l}\text { Extraction du beurre de cacao }=\ll \text { matière grasse } \\
\text { extraite à partir de fèves de cacao ou partie de fèves } \\
\text { de cacao» } \\
\text { Contrôle de qualité: la pureté du beurre de cacao est } \\
\text { déterminée, en analysant les pics obtenus en } \\
\text { chromatographie phase liquide: } 80 \% \text { des lipides } \\
\text { sont constitués par trois triacylglycérols: PO, POP } \\
\text { et SOS (acides P: palmitique, O: oléique, } \\
\text { S:stéarique) } \\
\text { Séparation des tourteaux }\end{array}$ \\
\hline 5 Pulvérisation & $\begin{array}{l}\text { Pulvérisateurs qui } \\
\text { réduisent en poudre les } \\
\text { tourteaux }\end{array}$ & Obtention de la poudre de cacao \\
\hline $\begin{array}{l}6 \text { Adjonction } \\
\text { d'additifs, de } \\
\text { sucre }\end{array}$ & $\begin{array}{l}\text { Mélange de la poudre de } \\
\text { cacao et des éventuels } \\
\text { additifs }\end{array}$ & Adjonction de sucre, de lécithine etc. \\
\hline \multicolumn{3}{|c|}{ Filière 2 : Obtention du chocolat } \\
\hline 7 Mélange brut & $\begin{array}{l}\text { Mélangeurs qui reçoivent } \\
\text { le sucre, le beurre de } \\
\text { cacao et éventuellement } \\
\text { le lait }\end{array}$ & Obtention d'une pâte homogène assez grossière \\
\hline $\begin{array}{l}8 \text { Broyage - } \\
\text { malaxage }\end{array}$ & $\begin{array}{l}\text { La pâte passe dans des } \\
\text { broyeurs }\end{array}$ & $\begin{array}{l}\text { Réduction de la granulation des particules solides à } \\
\text { une taille imperceptible à la langue et au palais } \\
\text { (inférieure à } 25 \text { microns) }\end{array}$ \\
\hline 9 Conchage & $\begin{array}{l}\text { Malaxage à chaud } 50 \text { à } \\
80{ }^{\circ} \mathrm{C} \text { durant } 24 \text { à } 72 \mathrm{~h} \text { en } \\
2 \text { phases : } \\
\text { - à sec : } 12 \mathrm{~h} \\
\text { - «liquide»: } 12 \mathrm{~h}\end{array}$ & $\begin{array}{l}\text { Conchage à sec: caramélisation du lactose et } \\
\text { formation de réaction de Maillard responsable des } \\
\text { arômes } \\
\text { Conchage «liquide»: adjonction de beurre de cacao } \\
\text { responsable de la plasticité, et du fondant } \\
\text { Pour des chocolats plus maigres : on ajoute plus de } \\
\text { lécithine }\end{array}$ \\
\hline 10 Tempérage & $\begin{array}{l}\text { Refroidissement de la } \\
\text { pâte }\end{array}$ & $\begin{array}{l}\text { Cristallisation du beurre de cacao sous sa forme } \\
\text { stable } \\
\text { Etape importante, car il faut porter à la température } \\
\text { précise de cristallisation du beurre de cacao qui sera } \\
\text { responsable du brillant du chocolat et de son } \\
\text { onctuosité. }\end{array}$ \\
\hline
\end{tabular}

Les produits étudiés ont été choisis en fonction de deux critères principaux que sont la présence de l'acrylamide dans un aliment, mise en évidence dans la littérature et son importance économique et sociale pour le consommateur. Les critères utilisés pour cette évaluation du risque étaient le type de traitement thermique (torréfaction, friture, grillade, cuisson au four), la durée et la température du traitement thermique, la présence de certains nutriments tels que les sucres réducteurs et les acides aminés et enfin, les conditions physico-chimiques de transformation des denrées.

\section{RESULTATS}

Analyse des méthodes de fabrication des aliments dérivés du cacao

Après la récolte des cabosses de cacao, la pâte, la poudre de cacao et le chocolat sont 


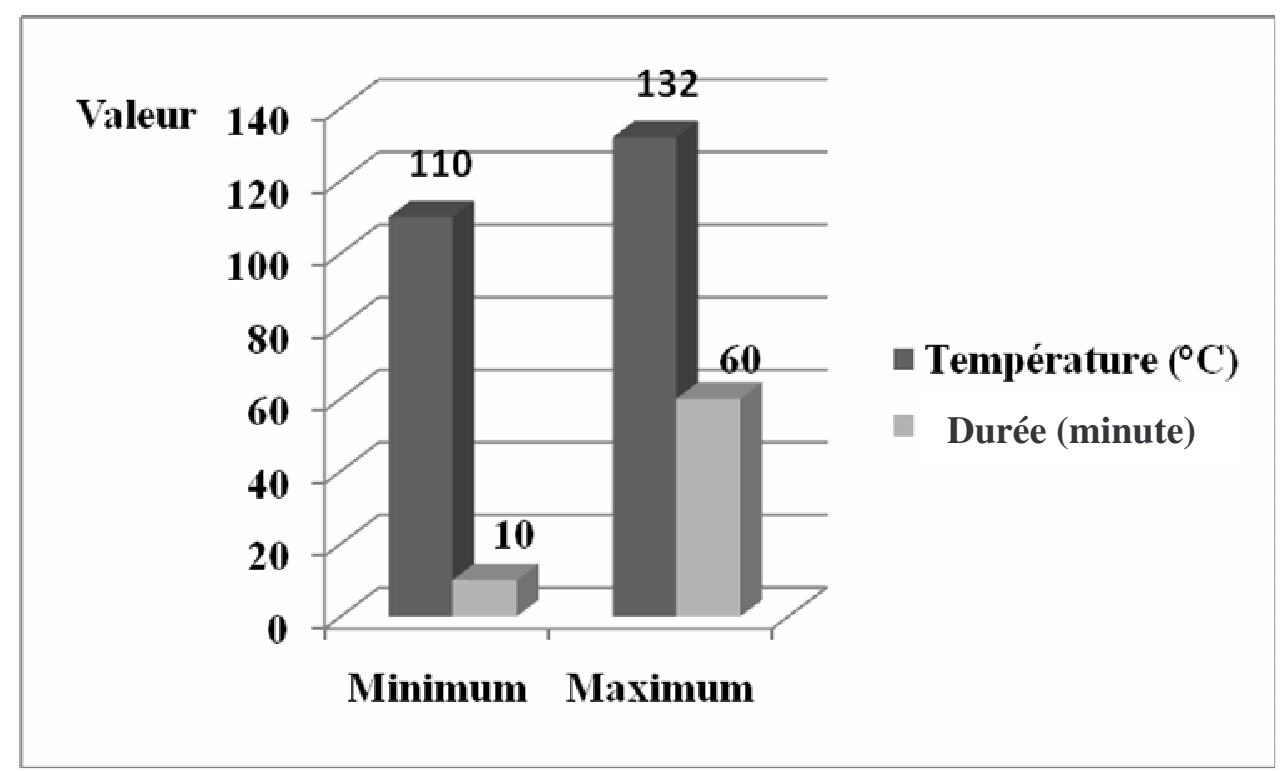

Figure 1: Température et durée de la torréfaction des produits dérivés du cacao.

obtenus par la transformation des fèves en plusieurs étapes (Tableaux 1 et 2). Au cours de celles-ci, la torréfaction des fèves est réalisée pendant une courte période de 10 minutes ou une longue période d'une heure. La température de torréfaction est comprise entre $110{ }^{\circ} \mathrm{C}$ et $132{ }^{\circ} \mathrm{C}$ (Figure 1). Différents traitements d'addition sont effectués pour améliorer la qualité technologique de la poudre de cacao et du chocolat (Tableau 3). Les étapes de transformation des fèves de cacao ont un intérêt technologique mais également des conséquences qui peuvent induire la formation de l'acrylamide.

Analyse des méthodes de fabrication des aliments dérivés du café

Après récolte, les deux cotylédons de la cerise sont extraits par des méthodes humide ou sèche.

\section{Méthode humide}

La cerise est cassée par dépulpage sous un filet d'eau. La pulpe obtenue est décomposée par fermentation dans des bassins d'eau pendant 2 à 5 jours. Le café est débarrassé de la pulpe fermentée par lavage puis séché à l'air ou dans des séchoirs artificiels à une température inférieure à $50^{\circ} \mathrm{C}$ pour éviter l'amertume du produit.

\section{Méthode sèche}

Les cerises sont directement séchées au soleil pendant 15-20 jours pour réduire la pulpe en poussière. Les grains sont ensuite débarrassés de l'endocarpe et torréfiés. La température du cylindre de torréfaction est de $200^{\circ} \mathrm{C}$ (Figure 2). L'arrivée d'une masse de matière froide la fait chuter à $120{ }^{\circ} \mathrm{C}$. Elle va remonter progressivement à $200{ }^{\circ} \mathrm{C}$ en 15 minutes. La composition chimique du café change faisant apparaître des arômes et modifiant la qualité gustative. Les constituants du café (sucres, acides organiques, protéines, alcaloïdes, graisses et minéraux) évoluent au contact de la chaleur. Ainsi, certains éléments disparaissent tandis que d'autres sont complexés. Le premier changement concerne la pigmentation. Le grain devient peu à peu ocre, brun et enfin quasi noir. Des réactions de Maillard se créent à partir de $160{ }^{\circ} \mathrm{C}$ et provoquent une harmonieuse interaction de protéines et de sucres qui aboutissent à des arômes. A l'approche de $200{ }^{\circ} \mathrm{C}$, les acides (précurseurs d'arôme), se combinent également pour former les différents arômes avant d'être détruits par carbonisation. Quand le café est torréfié à point, il est introduit dans un bac de refroidissement équipé de pales, de brosses et d'un puissant ventilateur. Ce 
Tableau 3: Traitements d'addition lors de la transformation des fèves de cacao.

\begin{tabular}{|c|c|}
\hline Traitements - produits d'addition & Intérêts \\
\hline $\begin{array}{l}\text { Alcalinisation : traitement alcalin par des produits } \\
\text { alcalinisants (carbonate de } \mathrm{K} \text { ou de } \mathrm{Mg} \text { ) avant la } \\
\text { torréfaction pour la production de poudre de cacao. }\end{array}$ & $\begin{array}{l}\text { Meilleure mouillabilité, dispersibilité de la } \\
\text { poudre de cacao. } \\
\text { Modification de la couleur: } \\
\text { - augmentation de l'intensité, } \\
\text { - possibilité d'orienter vers le brun- } \\
\quad \text { rouge } \\
\text { Modification de la flaveur: disparition de } \\
\text { l'acidité }\end{array}$ \\
\hline $\begin{array}{l}\text { Adjonction d'autres sucres que le saccharose } \\
\text { Adjonction autorisée < à } 5 \% \text { du poids total du } \\
\text { produit pour le glucose cristallisé, fructose, lactose, } \\
\text { maltose sans déclaration. } \\
\text { Si l'adjonction du glucose cristallisé est }>5 \% \text {, la } \\
\text { dénomination du produit doit être accompagnée de } \\
\text { la mention «avec glucose cristallisé» }\end{array}$ & $\begin{array}{l}\text { Adjonction de sucre réducteur pour } \\
\text { favoriser les réactions de Maillard. } \\
\text { Diminution du coût }\end{array}$ \\
\hline $\begin{array}{l}\text { Adjonction de polyols ou d'aspartam : } \\
\text { Réglementation relative aux produits } \\
\text { hypoglucidiques } \\
\text { Additifs autorisés et exemples : } \\
\text { Acidifiants: acide citrique E } 330 \text { etc. } \\
\text { Alcalinisants: carbonate de K, de Mg } \\
\text { Colorants: rouge de betterave E } 162 \text {, caramel E } 150 \\
\text { Emulsifiants: lécithines E } 322 \\
\text { Stabilisants: sorbitol E } 420 \text {, glycérol }\end{array}$ & Baisse de l'énergie \\
\hline
\end{tabular}

refroidissement est réalisé à l'air ou à l'eau grâce à une interruption rapide de la chaleur pour éviter la carbonisation des grains par auto-combustion.

\section{Analyse des préparations artisanales des aliments \\ Les féculents (igname, banane} plantain, patate douce, pomme de terre), les gâteaux (cakes) et la céréale (maïs) sont préparés selon 2 modes de cuisson que sont la cuisson à feux directs par grillade à l'air libre ou par gril au four et la cuisson dans l'huile (Tableau 4).

$\mathrm{Au}$ cours de la friture, les aliments sont immergés dans une grande quantité d'huile bouillante à une température de 140$180{ }^{\circ} \mathrm{C}$ pendant $15-25$ minutes. Ces aliments sont retirés de l'huile une fois dorés. Les aliments grillés à l'air libre sont mis en contact direct avec la chaleur du feu de bois pendant une dizaine de minutes. La température à laquelle ces aliments sont soumis est supérieure à $120{ }^{\circ} \mathrm{C}$ (Figure 3). Quant aux gâteaux, au cours de leur préparation, ils sont cuits au four à une température comprise entre $180{ }^{\circ} \mathrm{C}$ et $200{ }^{\circ} \mathrm{C}$. La durée de la cuisson varie entre 15 et 50 minutes (Figure 4).

\section{DISCUSSION}

La fabrication industrielle des produits dérivés du café et du cacao fait intervenir la torréfaction dont le degré varie selon l'origine des fèves, le type de café ou de chocolat à obtenir. La torréfaction est d'une grande importance pour la qualité du produit fini car c'est elle qui développe et affine l'arôme du produit par différentes réactions mise en œuvre au cours de ce processus. Cependant, des études ont montré que ces réactions sont prédominantes dans la synthèse de l'acrylamide dans les aliments (Becalski et al., 2003). 


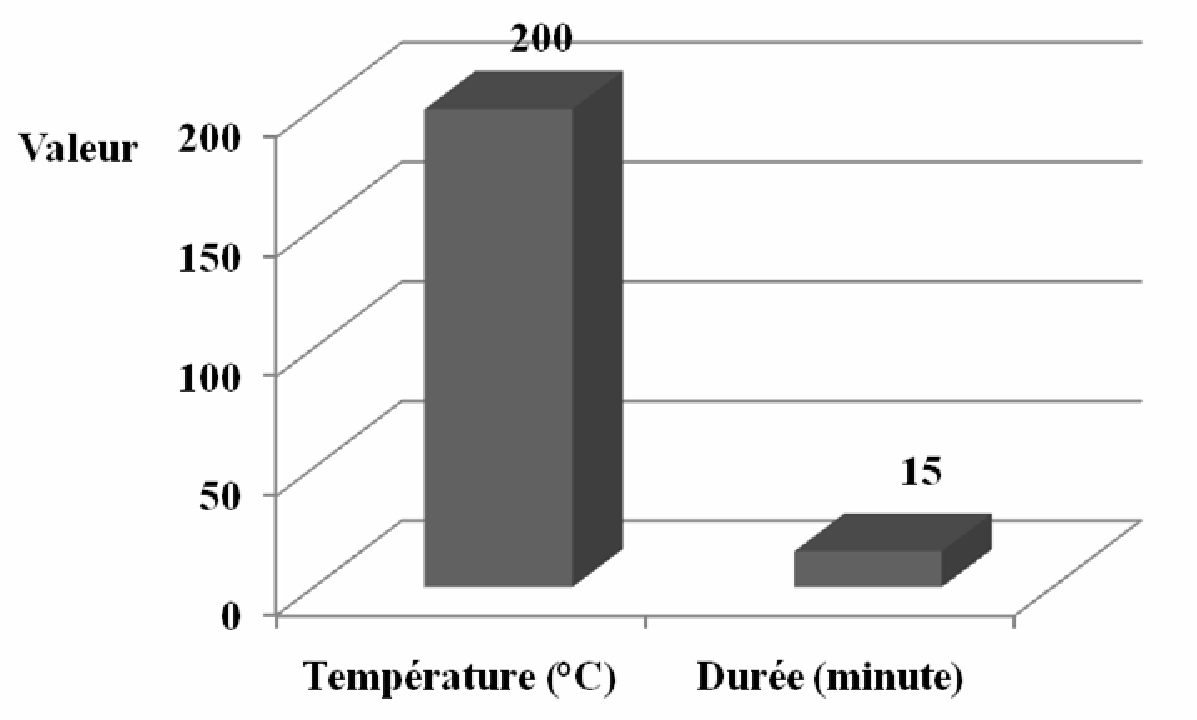

Figure 2: Température et durée de la torréfaction du produit dérivé du café.

Il apparaît que le café, le chocolat et la poudre de cacao, fabriqués par les entreprises enquêtées, pourraient présenter un risque d'apparition in situ d'acrylamide. En outre, des études réalisées en France par l'Agence Française pour la Sécurité Sanitaire des Aliments, au Canada par Santé Canada et aux Etats-Unis par la «Food and Drug Administration» ont montré que ces produits peuvent contenir des quantités non négligeables d'acrylamide (FAO/WHO, 2002). Ces quantités mesurées sont de l'ordre de $161-1300 \mu \mathrm{g} / \mathrm{kg}$ pour le café entre et de 60 $180 \mu \mathrm{g} / \mathrm{kg}$ pour le chocolat et les produits chocolatés.

En plus de l'acrylamide, les réactions de Maillard peuvent conduire à d'autres composés identifiés pour leur toxicité. Ces composés sont de la famille des furanoses principalement (l'hydroxyméthylfurfural, la furanosine, le furfuraldéhyde). Dans leur ensemble, ces substances sont néphrotoxiques à fortes doses et certaines ont aussi des effets mutagènes et cancérogènes (SKOG, 1993).

\section{Transformation du cacao \\ $\mathrm{Au}$ niveau des industries de transformations du cacao enquêtées, la}

méthode de fabrication fait apparaître plusieurs points critiques dans l'augmentation du risque de formation de l'acrylamide. Ces points sont le traitement d'alcalinisation, l'adjonction de sucres réducteurs, la température et la durée de torréfaction. Les deux derniers paramètres sont les plus déterminants dans la formation de l'acrylamide. En effet, des études (FAO/WHO, 2002, Gokmena et al., 2006) ont montré que l'acrylamide se forme dans les aliments à partir de $120^{\circ} \mathrm{C}$ et qu'une durée du traitement thermique longue en augmente la quantité formée.

Dans les méthodes de torréfaction, la température varie de $110{ }^{\circ} \mathrm{C}$ à $132{ }^{\circ} \mathrm{C}$ pendant 10 minutes à 1 heure. Le risque de formation de l'acrylamide est donc plus élevé pour les entreprises dans lesquelles la température et la durée de la torréfaction sont les plus élevées. Le traitement d'alcalinisation permet de faire disparaître l'acidité de la poudre de cacao et en modifier sa couleur. Cependant, il en résulte une augmentation du $\mathrm{pH}$ qui est un facteur précurseur de la formation de l'acrylamide (Biedermann et al., 2002). L'adjonction de sucres réducteurs qui est réalisée pour favoriser les réactions de 
Tableau 4: Aliments faits maison ou préparés sur le lieu de vente publique.

\begin{tabular}{|c|c|c|c|}
\hline $\begin{array}{l}\text { Groupes d'aliments } \\
\text { recensés }\end{array}$ & Aliments de base & Aliments préparés & Modes de cuisson \\
\hline Pâtisserie & $\begin{array}{l}\text { - Farine de blé } \\
\text { - Beurre } \\
\text { - Sucre et lait } \\
\text { - Levure chimique }\end{array}$ & - Gâteaux (cakes) & $\begin{array}{l}\text { - A feu direct } \\
\text { (au four) }\end{array}$ \\
\hline Céréales & - Maïs & - Maïs grillé & $\begin{array}{l}\text { - Grillade à feu direct (sur } \\
\text { du charbon de bois) }\end{array}$ \\
\hline $\begin{array}{l}\text { Aliments à base } \\
\text { d'amidon }\end{array}$ & $\begin{array}{l}\text { - Banane plantain } \\
\text { - Igname } \\
\text { - Pomme de terre } \\
\text { - Patate douce }\end{array}$ & $\begin{array}{l}\text { - Frites de banane } \\
\text { - Banane grillée } \\
\text { - Chips de banane } \\
\text { - Frites d'igname } \\
\text { - Igname grillée } \\
\text { - Frites de pomme de } \\
\text { terre } \\
\text { - Frite de patate douce }\end{array}$ & $\begin{array}{l}\text { - Friture à l'huile } \\
\text { - Grillade à feu direct (sur } \\
\text { du charbon de bois) }\end{array}$ \\
\hline
\end{tabular}

Maillard favorise également la formation de l'acrylamide (Stadler, 2003). Les aliments à base d'amidon sont ceux qui contiennent le plus d'acrylamide (FAO/WHO, 2002). Ainsi la fève de cacao qui contient 8 à $10 \%$ d'amidon pourrait permettre la synthèse de ce composé au cours des différentes réactions de traitement.

\section{Transformation du café}

Au niveau de l'entreprise de fabrication de café moulu enquêtée, la torréfaction est réalisée par la méthode traditionnelle à une température de $200{ }^{\circ} \mathrm{C}$. Cette méthode permet de développer l'arôme du café par les réactions de Maillard. Au cours de cette transformation, l'intensité et le degré de torréfaction varient. En effet, plus la torréfaction est courte, plus le café est acide et, plus la torréfaction est longue, plus le café gagne en douceur.

Parmi les différentes méthodes de torréfaction du café, la méthode traditionnelle favorise le plus la formation de l'acrylamide par les réactions de Maillard (Stadler, 2006). Ces réactions ne se produisent pas au cours de la torréfaction éclair et elles sont stoppées au moment où elles vont avoir lieu lors de la torréfaction rapide. Ainsi, ces deux dernières méthodes de torréfaction du café minimisent, voire empêchent la formation de l'acrylamide.
Le café et le cacao contiennent de l'asparagine dont le rôle de précurseur dans la formation de l'acrylamide a été prouvé (Biedermann et al., 2002) de même que celui des hydrates de carbones contenus dans le café $(58 \%)$.

\section{Préparation domestique des denrées alimentaires \\ L'enquête réalisée sur les denrées} alimentaires domestiques a montré que certains aliments de grande consommation sont préparés dans des conditions de cuisson non maîtrisées. Ces dernières pourraient favoriser la formation in situ de l'acrylamide. La grillade est le mode de cuisson qui développe le plus de benzopyrènes. Les croûtes formées lors des grillades ou fritures peuvent être le fait des réactions de Maillard qui augmentent le risque de formation de l'acrylamide (Stadler, 2006). De plus, la majorité des aliments recensés (banane plantain, igname, pomme de terre) sont riches en amidon. D'après la littérature (FAO/WHO, 2002), des quantités importantes d'acrylamide $(2000 \mu \mathrm{g} / \mathrm{kg})$ ont été trouvées dans les aliments riches en amidon.

La durée et la température des fritures et grillades varient d'une préparation à l'autre ce qui peut conduire à une augmentation du risque de formation de l'acrylamide. En effet, des études réalisées sur 


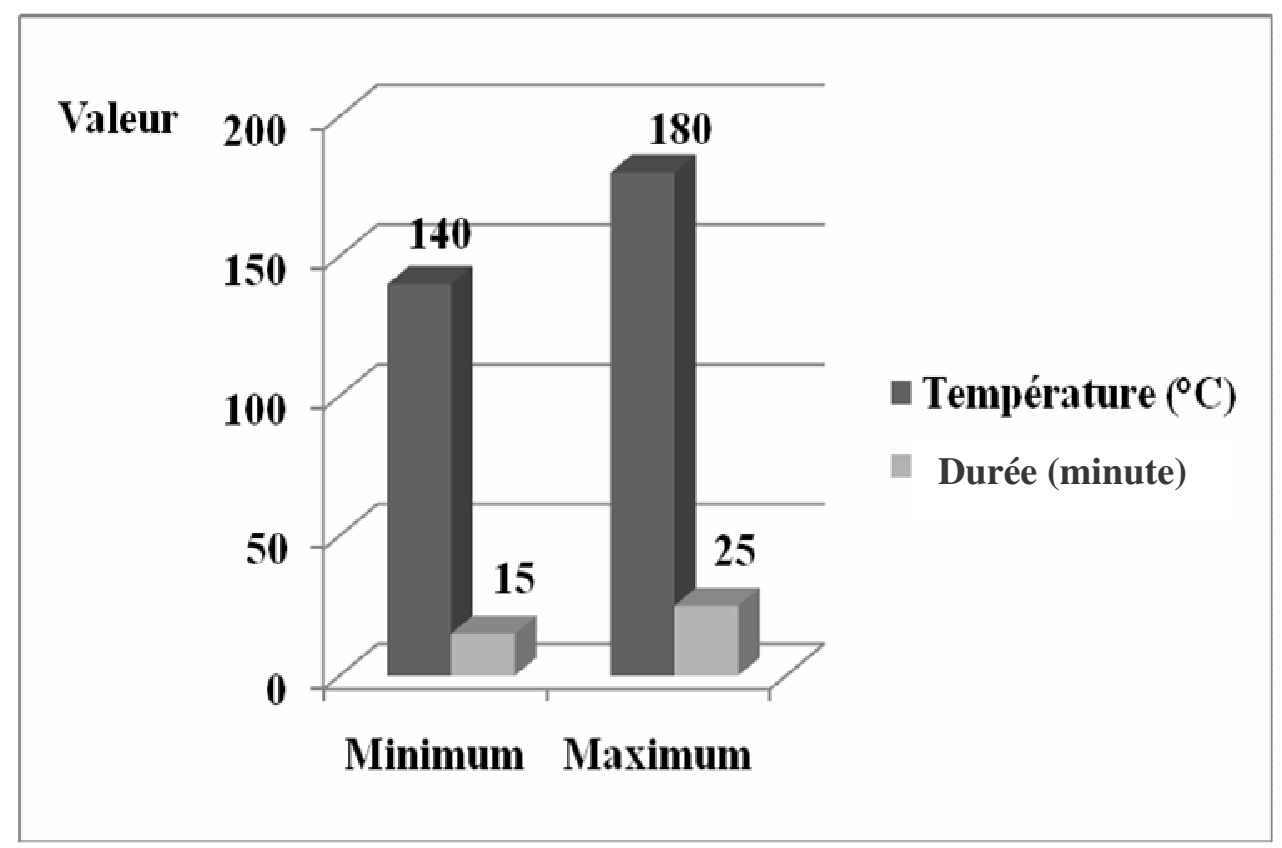

Figure 3 : Température et durée de cuisson des aliments frits ou grillés.

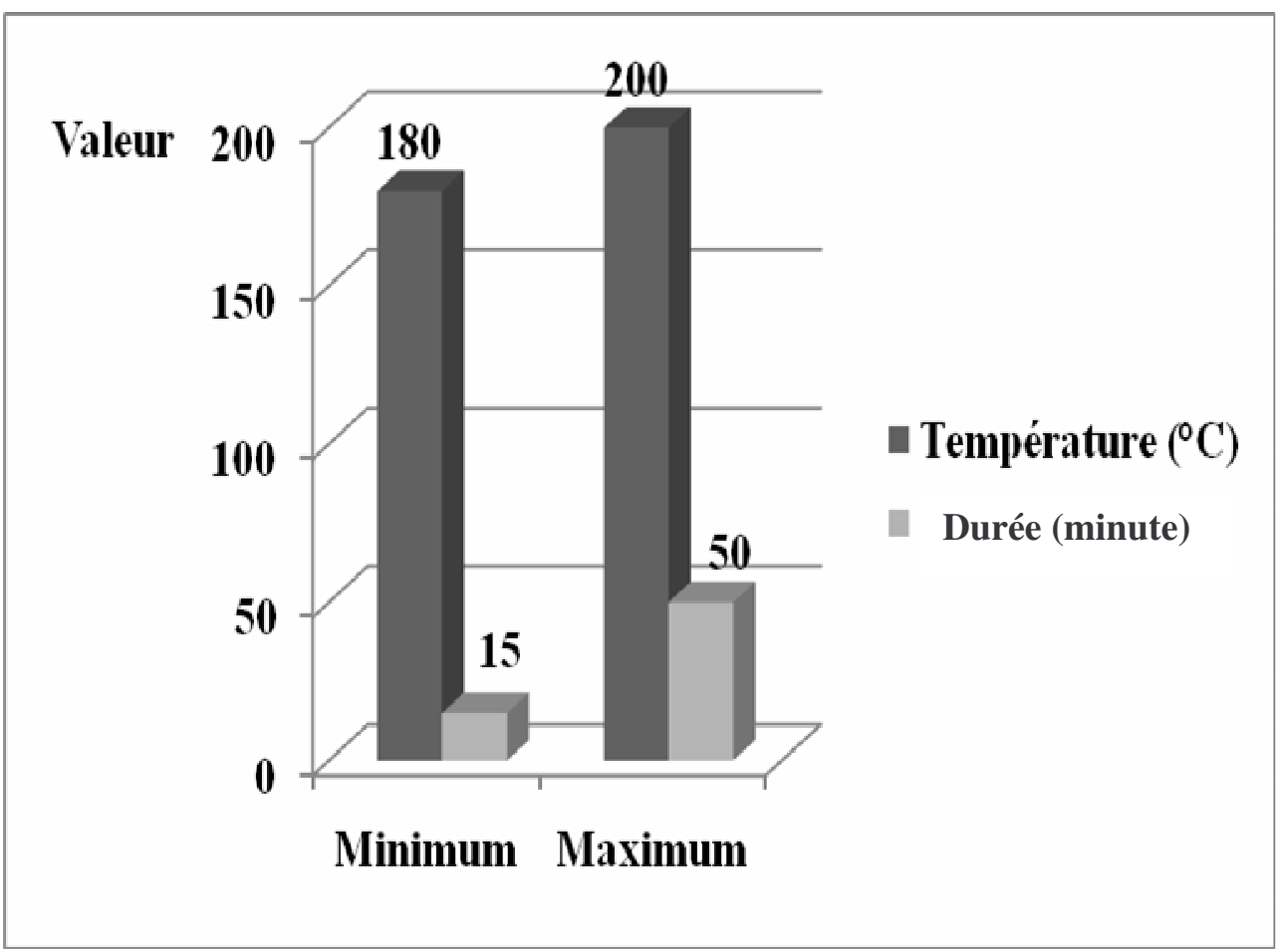

Figure 4: Température et durée de cuisson au four des gâteaux. 
la pomme de terre (Biedermann et al., 2002; Eriksson et al., 2007) ont montré le rôle de la température et de la durée de cuisson dans la formation de l'acrylamide à $180{ }^{\circ} \mathrm{C}$. Lorsque la durée de friture passe de 3 minutes à 5 minutes, la vitesse de formation de l'acrylamide est multipliée par 55 (Gokmena, 2006). De même, lorsque la température de friture passe de $160^{\circ} \mathrm{C}$ pendant 4 minutes à $180^{\circ} \mathrm{C}$ pendant 4 minutes, la vitesse de formation de l'acrylamide est multipliée par 12 (Biedermann et al., 2002).

L'acrylamide est une substance hydrosoluble et n'est donc pas présente dans la graisse ou l'huile. Lorsque les lipides utilisés pour la cuisson (huiles de friture) ou contenus dans les aliments sont soumis à des hautes températures, ils sont susceptibles de générer d'autres produits génotoxiques, tératogènes ou mutagènes par des mécanismes d'oxydation. Ces produits sont les Hydrocarbures Aromatiques Polycycliques (HAP), l'acroléine, l'oxycholestérol, la malonedialdéhyde et les polymères de péroxydes.

\section{Conclusion}

L'étude réalisée a révélé que, les températures, supérieures à $120{ }^{\circ} \mathrm{C}$, la durée des traitements thermiques industriels (torréfaction) ou domestiques (friture, grillade, cuisson au four) constituent les principaux facteurs de risque de formation de l'acrylamide. Pour les aliments industriels pris en compte dans notre étude, le risque est plus élevé pour le café dont la torréfaction est réalisée par la méthode traditionnelle alors que pour les aliments de préparations domestiques telles que les fritures de pommes de terre, le risque est élevé.

La prévention et la réduction du risque de formation de l'acrylamide sont possibles essentiellement en diminuant les concentrations de sucres réducteurs (glucose, saccharose), en changeant les conditions de transformation du café $(\mathrm{pH}<5)$, en diminuant la température et le temps de torréfaction au cours du traitement d'alcalinisation ou encore en faisant un traitement à l'asparaginase. Pour les aliments de préparation domestiques, la formation de l'acrylamide peut être minimisée en laissant les pommes de terre fraîchement découpées environ une heure dans l'eau avant de les cuire, en stockant les pommes de terre crues à l'abri de la lumière et à une température supérieure à $10{ }^{\circ} \mathrm{C}$, en évitant de carboniser les aliments cuits par grillade à feu direct ou encore, en faisant cuire les gâteaux jusqu'à l'obtention d'une couleur brun clair au lieu de brun foncé.

\section{REFERENCES BIBLIOGRAPHIQUES}

Becalski A, Lau BP-Y, Lewis D, SEAMAN SW. 2003. Acrylamide in Foods: Occurrence, Sources, and Modelling. Journal of Agricultural and Food Chemistry, 51: 802-808.

Bergmark E. 1997. Hemoglobin adducts of acrylamide and acrylonitrile in laboratory workers, smokers, and nonsmokers. Chemical Research in Toxicology, 10: 78-84.

Bergmark E. Calleman CJ, He F, Costa LG. 1993. Hemoglobin adducts in humans occupationally exposed to acrylamide. Toxicology and Applied Pharmacology, 120: 45-54.

Biedermann M, Noti A, Biedermann-brem S, Mozzetti V, Grob K. 2002. Experiments on acrylamide formation and possibilities to decrease the potential of acrylamide formation in potatoes. Mitteilungen aus Lebensmitteluntersuchung und Hygiene, 93: 668-687.

Bull RJ, Robinson M, Laurie RD, Stoner GD, Greisiger E, Meier JR, Stober J, 1984. Carcinogenic effects of acrylamide in Sencar and A/J mice. Cancer Research, 44: 107-111.

Edwards PM. 1976. The insensitivity of the developing rat foetus to the toxic effects of acrylamide. Chemico-biological Interactions, 12: 13-18.

Eriksson AS, Karlsson BP, Tornqvist M. 2007. Measurement of evaporated acrylamide during heat treatment of food and other biological materials. Food Science and Technology, 40: 706-712.

European Commission, 2002. New findings regarding the presence of acrylamide in food: Opinion of the Scientific Committee on Food. European commission: Scientific Committee on Food report SCF/CS/CNTM/CONT/4, 16 pages, Brussel, Belgium.

FAO/WHO, 2002. Consultation on the Health Implications of Acrylamide in Food. FAO/WHO report: 25-27 June 200212 pages, Geneva, Switzerland. 
Gokmena V, Palazoglub TK, Senyuvac HZ. 2006. Relation between the acrylamide formation and time-temperature history of surface and core regions of French fries. Journal of Food Engineering, 77: 972-976.

Hashimoto K, Aludridge NW. 1970. Biochemical studies on acrylamide, a neurotoxic agent. Biochemical Pharmacology, 19: 2591-2604.

International Agency For Research ON Cancer (IARC). 1994. Acrylamide: IARC monographs on the evaluation of the carcinogenic risk of chemicals to humans. IARC Some Industrial Chemicals, Lyon, France, 60: 389-433.

Johnson KA, Gorzinski SJ, Bodner KM, Campbell R, Wolf C, FRiedman M, Mast R. 1986. Chronic toxicity and oncogenicity study on acrylamide incorporate in the drinking water of Fischer 344 rats. Toxicology and Applied Pharmacology, 85: 154-168.

Marchetti F, Lowe X, Bishop J, Wyrobek AJ. 1997. Induction of chromosomal aberrations in mouse zygotes by acrylamide treatment of male germ cells and their correlation with dominant lethality and heritable translocations. Environmental and Molecular Mutagenesis, 30: 410-417.

Miller MJ, Carter DE, Sipes IG. 1982. Pharmacokinetics of acrylamide in
Fischer-344 rats. Toxicology and Applied Pharmacology, 63: 36-44.

Ono H, Chuda Y, Ohnishi-kameyama M, Yada h, Ishizaka M, Kobayashi $\mathrm{H}$, Yoshida M. 2003. Analysis of acrylamide by LC-MS/MS and GC-MS in processed Japanese foods. Food Additives and Contaminants, 20: 215220.

Rosen J, Hellenas K. 2002. Analysis of acrylamide in cooked foods by liquid chromatography tandem mass spectrometry. The Analyst, 127: 880882.

Shiraishi Y. 1978. Chromosome aberrations induced by monomeric acrylamide in bone marrow and germ cells of mice. Mutation Research, 57: 313-324.

Skog K. 1993. Cooking procedures and food mutagens: A literature review. Food and Chemical Toxicology, 31: 655-675.

Smith MK, Zenick H, Preston RJ, George EL, Long RE. 1986. Dominant lethal effects of subchronic acrylamide administration in the male Long-Evan rat. Mutation Research., 173: 273-278.

Stadler RH. 2003. Understanding the formation of acrylamide and other Maillard-derived vinylogous compounds in foods., European Journal of Lipid Science and Technology, 105: 199-200. 